\title{
NIVELES DE GLOMALINA EN UN SUELO CON PLANTACIONES DE PINO RADIATA (Pinus radiata D. Don.) Y DISTINTOS TIEMPOS DE PLANTACION
}

\author{
Diana N. EFFron; Cristina QuinTEROS; Silvia I. CATÁN; Gabriela C. SARTI y Rosa L. DEFRIERI(1)
}

\begin{abstract}
RESUMEN: Los hongos micorrízicos arbusculares (HMA) son microorganismos simbiontes que forman asociaciones mutualísticas con raíces de la mayoría de las plantas superiores. Los HMA producen glomalina que contiene un alto porcentaje de carbono y su contenido puede usarse como un indicador de los efectos del cambio de uso del suelo. El objetivo de este estudio fue evaluar el contenido de glomalina total (GT) y glomalina fácilmente extraíble (GFE) en un suelo andisol de la provincia de Chubut, Argentina, bajo la influencia de la especie forestal Pinus radiata D. Don. en dos parcelas con diferentes tiempos de plantación, una con 50 años (P50) y otra con 20 años (P20); y vincular dichos niveles con el carbono orgánico, las propiedades biológicas y bioquímicas del suelo. Los resultados mostraron que los valores de los parámetros dosados dependieron del tiempo de plantación, resultando significativamente mayores en el suelo con la plantación de 50 años. Los mayores valores del $\mathrm{C}$ de respiración y de las actividades enzimáticas en el suelo bajo P50 podrían vincularse con los mayores niveles de glomalina hallados, ya que la misma, al influir en el mejoramiento de los agregados del suelo y generar un aumento en el contenido de $\mathrm{C}$ orgánico, causaría en el mismo un incremento en la actividad microbiana del suelo.
\end{abstract}

ABSTRACT: Arbuscular mycorrhizal fungi (AMF) are microorganisms that form mutualistic symbiotic associations with roots of most of plants. Glomalin produced by AMF contains a high percentage of carbon and it can be used as an indicator of the effects of changing land use. The aim of this study was to evaluate the contents of total glomalin (GT) and easily extractable glomalin (EEG) in an andisol of Chubut province, Argentina, under influence of Pinus radiata D. Don. in two plots with different plantation time, one of 50 years (P50) and the other of 20 years (P20) and linking to organic carbon and soil biological and biochemical properties. The results showed that the values of the parameters were influenced by plantation time. Values obtained were significantly higher in the plot P50. The highest values of respiration carbon and enzyme activities in soil under P50 could be linked to higher levels of glomalin, because this glycoprotein improve soil aggregates and generate an increase in the content of organic carbon and then it could increase microbial activity in this soil.

Palabras claves: glomalina, Pinus radiata D. Don., tiempo de plantación, carbono de respiración, actividades enzimáticas.

Key words: glomalin, Pinus radiata D. Don., plantation time, respiration carbon, enzyme activities.

\section{INTRODUCCIÓN}

Los hongos micorrízicos arbusculares (HMA) son microorganismos simbiontes que forman asociaciones mutualísticas con raíces de la mayoría de las plantas superiores aumentando la tasa fotosintética. Los HMA contribuyen a la nutrición mineral de la planta hospedera, protegen frente a estreses tanto bióticos como abióticos, valorándose en la actualidad su rol en el almacenamiento de C del suelo (Seguel et al., 2008).

(1) Cátedra de Química General e Inorgánica. Facultad de Agronomía, Universidad de Buenos Aires. Avda. San Martín 4453, CABA. E-mail: effron@agro.uba.ar 
Además, los HMA producen glomalina, una glicoproteína insoluble de elevado peso molecular. El comportamiento recalcitrante de la misma y su característica hidrófoba, que protege a las hifas de las pérdidas de nutrientes y agua, sugieren que es una biomolécula muy estable con lenta velocidad de degradación y una vida media entre 6 y 42 años, dependiendo del ecosistema suelo, condiciones ambientales y manejos. La estructura química de la glomalina contiene un alto porcentaje de carbono, llegando a representar hasta $52 \%$ del carbono total en suelos orgánicos, el cual es incorporado al suelo mediante la descomposición de los propágulos fúngicos. Borie et al. (2000) y Arriagada et al. (2004), sugieren que las hifas de hongos ectomicorrízicos estabilizan los agregados en diversos suelos forestales. Según Rillig et al. (2003) la glomalina puede usarse como indicador de los efectos del cambio de uso del suelo.

Estas proteínas fúngicas pueden extraerse desde el suelo o desde las hifas de hongos, obteniéndose distintas fracciones según el procedimiento de extracción. Entre ellas la fracción glomalina fácilmente extraíble (GFE) que es considerada un material recientemente depositado en el suelo y de naturaleza muy lábil y otra fracción la glomalina total (GT) que corresponde a la proteína fuertemente unida a las partículas del suelo (Lovelock et al., 2004).

Actualmente, crece el interés por correlacionar los niveles de glomalina en el suelo con otros parámetros físico-químicos y biológicos (Treseder y Turner, 2007; Seguel et $a l, 2008)$ existiendo aún pocos estudios al respecto.

El objetivo de este estudio fue evaluar el contenido de glomalina total (GT) y glomalina fácilmente extraíble (GFE) en un suelo andisol de la provincia de Chubut, Argentina, bajo la influencia de la especie forestal Pino radiata (Pinus radiata D. Don.) en dos parcelas con diferentes tiempos de plantación, una con 50 años (P50) y otra con 20 años (P20), vinculándolos con el carbono orgánico, las propiedades biológicas y bioquímicas del suelo.

\section{MATERIALES Y MÉTODOS}

El estudio se instaló en la Estación Forestal del INTA, Trevelín, provincia de Chubut, ubicada en la Colonia 16 de Octubre, Futaleufú, Latitud $43^{\circ}$ Sur y Longitud $71^{\circ} 31^{\text {' }}$ Oeste, altitud 470 m s.n.m; ocupa 3020 ha y sus suelos están clasificados como Andisoles (Colmet Dâage et al., 1988).

Se seleccionaron al azar 10 árboles de 2 parcelas con una superficie de 2 ha cada una con Pino radiata (Pinus radiata D.Don.) con diferentes tiempos de plantación: una con 50 años (P50) y la otra con 20 años (P20). El suelo de las plantaciones estaba cubierto por una capa de acículas no observándose la presencia de herbáceas ni arbustivas. Los árboles seleccionados presentaban buen estado sanitario y portes similares. Se tomaron muestras superficiales $(0$ a $10 \mathrm{~cm}$ ) de suelo (previo despeje del material vegetal superficial, y a una misma distancia del tronco de los árboles seleccionados). Debajo de cada uno de los árboles se tomaron 4 muestras de las cuales se hizo una muestra compuesta por árbol. 
Sobre las mismas se efectuaron las siguientes determinaciones: glomalina total (GT), glomalina fácilmente extraíble (GFE), carbono de respiración, carbono orgánico, actividades de las enzimas ß-glucosidasa, fosfatasa ácida y proteasas y fósforo disponible.

Glomalina total (GT): se determinó mediante extracciones sucesivas con citrato de sodio $50 \mathrm{mM}$ a pH 8,0 y autoclavado por 1 hora hasta la desaparición del color pardo rojizo característico de la glomalina y posterior determinación espectrofotométrica de acuerdo al método de Bradford para proteínas (Lovelock et al., 2004).

Glomalina fácilmente extraíble (GFE): se extrajo con citrato de sodio $20 \mathrm{mM}, \mathrm{pH}$ 7,0 y autoclavado por 30 minutos con posterior determinación espectrofotométrica con igual metodología que la glomalina total (Wright y Upadhyaya, 1998; Morales et al., 2005).

Carbono de respiración microbiana: se midió el $\mathrm{CO}_{2}$ liberado durante la incubación del suelo, el que es retenido por una solución de $\mathrm{NaOH}$ y posterior valoración del $\mathrm{NaOH}$ remanente (Anderson, 1982).

Carbono orgánico: se determinó por el método de Walkley y Black (Nelson y Sommers, 1982).

Actividad fosfatasa ácida y actividad $\beta$-glucosidasa: se determinaron por incubación de las muestras de suelo con los sustratos p-nitrofenil fosfato de sodio (PNP) y pnitrofenil glucósido (PNG) respectivamente, a temperatura y $\mathrm{pH}$ óptimos y posterior lectura espectrofotométrica a $410 \mathrm{~nm}$ del p-nitrofenol liberado (Dick et al.,1996).

Actividad de proteasas: se determinó por incubación del suelo durante 2 hs a $50^{\circ} \mathrm{C}$, con caseína como sustrato a pH 8,1. La tirosina liberada se determinó por colorimetría con el reactivo de Folin-Ciocalteau (Dilly y Munch, 1996).

Fósforo disponible: se evaluó según la técnica de Bray-Kurtz No. 1 (Olsen y Sommers, 1982).

\section{Análisis estadístico}

Los datos para las variables estudiadas fueron analizados estadísticamente mediante un análisis de varianza de una vía perteneciente a un diseño completamente aleatorizado entre tratamientos correspondientes a distintos tiempos de plantación. Las diferencias entre medias de tratamientos fueron determinadas mediante el test de Tukey $(p<0,05)$. Se utilizó el programa estadístico INFOSTAT /PROFESIONAL ® Versión 1.1- Universidad de Córdoba.

\section{RESULTADOS}

Los niveles de glomalina (GT y GFE) resultaron significativamente mayores ( $\mathrm{p}<$ 0,05) en el suelo con el Pino (P50) (Fig. 1) al igual que el carbono orgánico y de respiración y las actividades de las enzimas medidas (Tablas 1 y 2).

Los valores de GT y GFE fueron mayores a los obtenidos por Bedini et al. (2007) quienes hallaron valores de GT entre 2 y $10 \mathrm{mg} \cdot \mathrm{g}^{-1}$ y entre 0,2 y $1,5 \mathrm{mg} . \mathrm{g}^{-1}$ de GFE para suelos sometidos a distintos usos (agricultura, bosques no manejados y pastizales). 


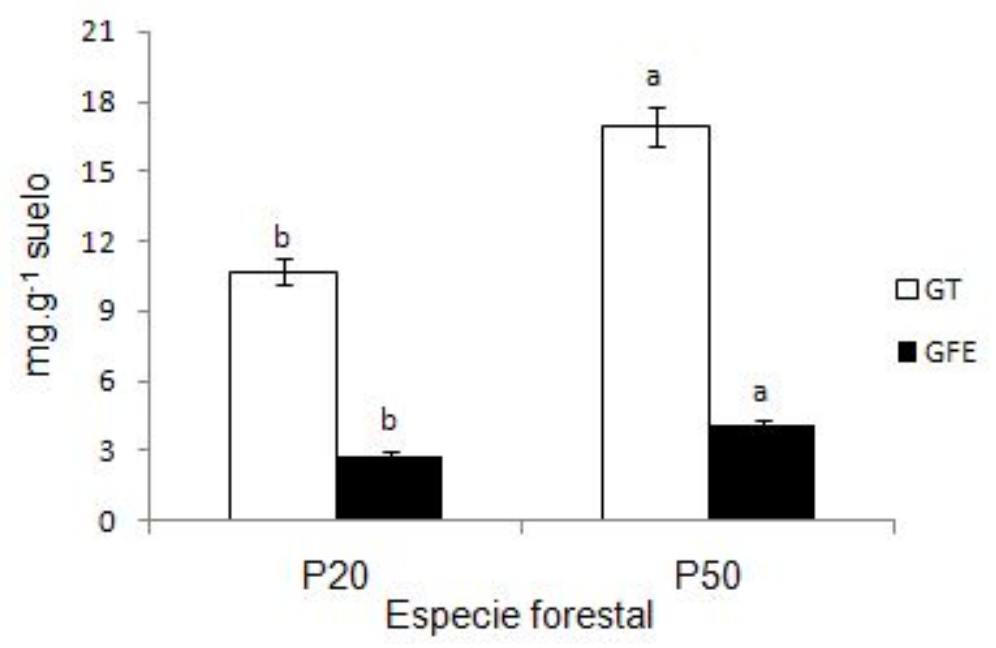

Fig. 1: Valores medios de glomalina total (GT) y glomalina fácilmente extraíble (GFE) en el suelo debajo de Pino (Pinus radiata D. Don.) de P20 (20 años de plantación) y P50 (50 años de plantación). Letras diferentes indican diferencias significativas $(\mathrm{p}<0,05)$ entre tiempos de plantación.

Tabla 1: Valores medios de $\mathrm{C}$ orgánico, $\mathrm{C}$ de respiración y de $\mathrm{P}$ disponible en el suelo bajo un bosque de Pino radiata (Pino Radiata D. Don.) con 20 años (P20) y 50 años de plantación (P50). Letras diferentes indican diferencias significativas $(\mathrm{p}<0,05)$ entre tiempos de plantación.

\begin{tabular}{|c|c|c|c|}
\hline & $\begin{array}{l}\text { C orgánico } \\
\left({\mathrm{g} . \mathrm{kg}^{-1}}_{\text {suelo }}\right)\end{array}$ & 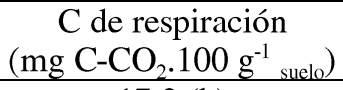 & 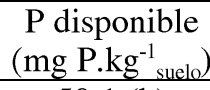 \\
\hline P20 & 20,0 (b) & 17,3 (b) & 50,1 (b) \\
\hline $\mathrm{P} 50$ & 67,3 (a) & 33,5 (a) & 37,3 (a) \\
\hline
\end{tabular}

Tabla 2: Valores medios de las actividades enzimáticas de la fosfatasa ácida, $\beta$-glucosidasa y proteasas en el suelo bajo la influencia de la especie Pino radiata (Pinus Radiata D. Don.) con 20 años (P20) y 50 años de plantación (P50). Letras diferentes entre filas indican diferencias significativas $(\mathrm{p}<0,05)$ entre tiempos de plantación.

\begin{tabular}{cccc}
\hline & Fosfatasa ácida & B-glucosidasa & Proteasas \\
\hline & \multicolumn{2}{c}{$\left(\mathrm{mg}\right.$ paranitrofenol $\left.\mathrm{kg}^{-1} \mathrm{~h}^{-1}\right)$} & $\left(\mathrm{mg}\right.$ tirosina $\left.\mathrm{kg}^{-1} \mathrm{~h}^{-1}\right)$ \\
\hline P20 & $450,9(\mathrm{~b})$ & $164,2(\mathrm{~b})$ & $354,6(\mathrm{~b})$ \\
P50 & 824,7 (a) & $184,5(\mathrm{~b})$ & $503,2(\mathrm{a})$ \\
\hline
\end{tabular}

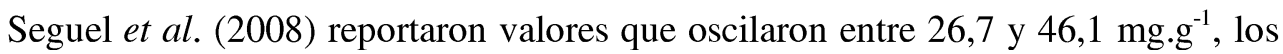
que corresponden a un andisol de un bosque mixto de olivillo para GT y entre 9 y 25 mg. $\mathrm{g}^{-1}$ para GFE. Estos investigadores sugieren que los niveles de glomalina en ecosistemas forestales serían superiores al de los agrosistemas, posiblemente debido a que el incremento en la velocidad de descomposición de los diversos propágulos de HMA presentes en los suelos bajo bosques, favorecen la llegada de la glomalina a la fracción húmica del suelo. 
Yadav y Tarafdar (2004) señalan que la edad fisiológica de los árboles tiene directa incidencia en la actividad de las enzimas, especialmente en la fosfatasa ácida la cual aumenta con la edad de los árboles, en concordancia con los resultados de este trabajo. Resultados similares obtuvieron Alvear et al. (2007 a) quienes concluyeron que la mayoría de las actividades biológicas fueron influenciadas por las etapas sucesionales evaluadas, siendo significativamente mayores en la tapa de fustal (árboles maduros) que en la etapa de brinzal (árboles jóvenes). Por el contrario, Alvear et al. (2007 b) comparando un bosque maduro con uno secundario en el sur de Chile, encontraron valores mayores en las actividades biológicas evaluadas del bosque secundario vinculando este comportamiento con la mayor humedad determinada en el mismo, condición que favorece el desarrollo y proliferación de la biomasa microbiana. Rebottaro et al. (2010) trabajando con plantaciones de Pinus elliottii en suelos arenosos concluyeron que el contenido de carbono orgánico del suelo aumentó con la edad de la plantación. Por otra parte, Gómez et al. (2008) trabajando con plantaciones de Pinus caribaea de 3 y 29 años encontraron mayor contenido de C orgánico y de respiración en el suelo bajo la influencia de la plantación de 29 años con respecto a una sabana considerada como control. El incremento en la concentración de carbono en este rodal, los autores lo asociaron al mayor contenido de acículas acumuladas en la superficie del suelo mineral, al reducido ciclaje y al largo período de residencia de la materia orgánica en estas plantaciones.

Por otro lado, en este trabajo se encontraron que los contenidos de $\mathrm{P}$ disponible (Tabla 1) variaron significativamente $(\mathrm{p}<0,05)$ siendo los menores valores para el suelo en (P50). Alvear et al. (2007 a) explicaron que cuanto mayores son los niveles de la actividad de la fosfatasa, para la plantación con más edad, se genera una retro inhibición en la liberación de $\mathrm{P}$ disponible que explica los menores valores de $\mathrm{P}$ obtenidos.

Los mayores valores del $\mathrm{C}$ de respiración y de las actividades enzimáticas vinculados a los ciclos del C, P y N en el suelo en P50 encontrados en este trabajo, podrían vincularse con los mayores niveles registrados de glomalina, ya que la misma al influir en el mejoramiento de los agregados del suelo y generar un aumento en el contenido de $\mathrm{C}$ orgánico, incrementarían la actividad de los microorganismos del suelo.

\section{CONCLUSIONES}

De los resultados obtenidos se concluye que los niveles de glomalina podrían utilizarse como otro indicador de calidad de suelos además del carbono orgánico, las propiedades biológicas y bioquímicas, para evaluar el efecto de plantaciones forestales sobre el suelo a diferentes edades. 


\section{AGRADECIMIENTOS}

Este trabajo fue financiado por el proyecto de Ciencia y Técnica de la Universidad de Buenos Aires (Código 20020090100087BA) de la programación científica de los años 2010-2012.

\section{BIBLIOGRAFÍA}

AlVEAR, M.; F. ReYes y A. Morales, 2007 b. Actividad biológica y agregados estables del agua en dos tipos de formaciones vegetales de un bosque templado del centro-sur de Chile con perturbación antrópica. Ecología Austral, 17:113-122.

Alvear, M.; C. Urra; R. Huaiquilao; M. Astorga y F. Reyes, 2007 a. Actividades biológicas y estabilidad de agregados en un suelo del bosque templado chileno bajo dos etapas sucesionales y cambios estacionales. Revista Ciencia del suelo Nutrición Vegetal, 7, 3: 38-50.

ANDERSON, T., 1982. Soil respiration. Pp. 841-845. In: Page et al. (Eds.): Methods of soil Analysis Agrono$m y$. ASA y SSSA. Madison, Wisconsin,USA.

ARriagada, C.A; M.A. Herrera; I. Garcia-Romera y J.A. OCAmpo, 2004. Tolerance to Cd of soybean (Glycine max) and eucalyptus (Eucalyptus globulus) inoculated with arbuscular mycorrhizal and saprobe fungi. Symbiosis, 36: 285-299.

Bedini, S.; L. Avio; E. ARgeSe y M. GiovannetTi, 2007. Effects of long-term land use on arbuscular mycorrhizal fungi and glomalin-related soil protein. Agriculture, Ecosystems and Environment, 120: 463-466.

Borie, F.; I. Rubio; A. Morales y C. CAstillo, 2000. Relación entre la densidad de hifas de hongos micorrizógenos arbusculares y producción de glomalina con las características físicas y químicas del suelo bajo cero labranza. Revista chilena de Historia natural, 73:749-756.

Colmet DÂage, F; A. Marcolin; C. López; A. Lanciotti; J. Ayesa; D. Bran; E. Andenmatten; P. Broquen; J. Girardin; G. Cortés; J. IrISARRi; E. Besoain; E. SadZawKa; G. SEPÚlveda; S. MASSARO; G. Millot y P. Bouleau, 1988. Características de los suelos derivados de cenizas volcánicas de la cordillera y precordillera del norte de la Patagonia. Bariloche. Convenio INTA-ORSTOM S. C. de Bariloche, Río Negro. 167 p.

Dick, R.P; D.P. BRAKWELl y R.F. TuRCO, 1996. Soil enzyme activities and biodiversity measurements as integrative micribiological indicators. Pp. 247-271. En; Doran J y Jones A (eds.): Methods for Assessing Soil Quality. SSSA Spec. Publ. Número 49.

DiLly, O. y J.C. MuNCH, 1996. Microbial biomass content, basal respiration and enzyme activities during the course of decomposition of leaf litter in a black alder (Alnus glutinosa(L.) Gaertn) forest. Soil Biological. Biochemistry, 28:1073-1081.

GómEZ, Y.; J. PAOLINI y R.M. HERNÁNDEZ, 2008. Sustitución de la sabana nativa con plantaciones de Pinus caribaea (Pinaceae) en Venezuela: efecto sobre parámetros indicadores de cambios en el carbono del suelo. Revista de Biología Tropical, 56: 2041-2053.

Lovelock, C.; S. Wright; D.A. Clark y R.W. Ruess, 2004. Soil stocks of glomalin produced by arbuscular mycorrhizal fungi across a tropical rain forest landscape. Journal Ecology, 92: 278-287.

Morales, A.; R.W. CASTiLlo; R. Rubio; F. Borie y J.L. RouAnEt, 2005. Niveles de glomalina en suelos de dos ecosistemas del sur de Chile. Revista Ciencia del Suelo Nutrición Vegetal, 5: 37-45.

NELSON, D.W. y L.E. SOMMERS, 1982. Total carbon, organic carbon and organic matter. Pp. 539-579. In: Page, Miller y Keeny (eds.): Methods of Soil Analysis, 2nd edn, Part 2. American Society of Agronomy. Madison.Wisconsin. USA. 
Olsen, S. y R.L.E. Sommer, 1982. Phosphorus. Pp. 403-430. In: Page, Miller y Keeny (eds.): Methods of soil analysis. Part 2. 2nd edn. American Society of Agronomy. Madison. Wiscosin. USA,.

Rebottaro, S.; D. Cabrelli y E. Rienzi, 2010. Changes in mineral and organic soil in pine stands with different age and management. The International Forestry Review. Ed. Commonwealth Forestry Association, 12: 51 .

RILlig, M.; P.W. RAMSEY; S. MoRris y E.A. PAUL, 2003. Glomalin, an arbuscular mycorrhizal fungal soil protein, responds to soil-use change. Plant and Soil, 253: 293-299.

Seguel, A.; R. Rubio; R. CARrillo; A. EsPinosa y F. Borie, 2008. Niveles de glomalina y su relación con características químicas y biológicas del suelo (andisol) en un relicto de bosque nativo del sur de Chile. Bosque (Valdivia), 9: 11-22.

TreSEDER, K.K. y K.M. TuRner, 2007. Glomalin in ecosystems. Soil Science Society of America Journal, 71, 4: 1257-1266.

WRIGHT, S.F y A. UPADHYAYA, 1998. A survey of soils for aggregate stability and glomalin, a glycoprotein produced by hyphae of arbuscular mycorrhizal fungi. Plant Soil 198: 97-107.

YADAV, B.K y J.C. TARAFDAR, 2004. Phytase activity in the rhizosphere of crops, trees and grasses under arid environment. Journal of Arid Environment, 58: 285-293. 